\title{
Angular anisotropy parameters and recoil-ion momentum distribution in two-photon double ionization of helium
}

\author{
A. S. Kheifets, ${ }^{1, *}$ I. A. Ivanov, ${ }^{1, \dagger}$ and Igor Bray ${ }^{2}$ \\ ${ }^{1}$ Research School of Physical Sciences and Engineering, The Australian National University, Canberra ACT 0200, Australia \\ ${ }^{2}$ ARC Centre for Matter-Antimatter Studies, Curtin University, GPO Box U1987, Perth, WA 6845, Australia
}

(Received 18 May 2007; published 28 August 2007)

\begin{abstract}
We present convergent-close-coupling (CCC) calculations of the angular anisotropy parameters $\beta_{2}, \beta_{4}$ and the recoil ion momentum distribution $d \sigma / d p$ in two-photon double ionization (TPDI) of helium. In a stark contrast to single-photon double ionization (SPDI), where the $\beta_{2}$ parameter varies widely changing the angular distribution from isotropic to nearly dipole for slow and fast photoelectrons, respectively, the $\beta$ parameters for TPDI show very little change. The angular distribution of the recoil ion is fairly isotropic in TPDI as opposed to a strong alignment with the polarization of light in SPDI.
\end{abstract}

DOI: 10.1103/PhysRevA.76.025402

PACS number(s): 42.50.Hz, 32.80.Fb

There has been a considerable amount of work done recently on two-photon double ionization (TPDI) of He. This work was largely driven by a rapid advancement in experimental techniques. The free electron laser at Hamburg (FLASH) started delivering vacuum ultraviolet photons at intensities sufficient for observing various multiphoton ionization phenomena. On the theoretical side, a wide range of methods was applied to the TPDI problem during the past decade. There have been several reported calculations of the total integrated cross section of TPDI of He at various photon energies [1-7]. Although numerical values of the cross sections varied depending on the theoretical model and assumed characteristics of the laser field, there appeared some consensus between several calculations (see Hu et al. [7] for detail). This consensus, however, was challenged recently as can be seen from the latest reports [8-10].

In contrast, the data on angular correlation pattern in twoelectron continuum are more conciliatory. There is at least some agreement between the fully resolved triply differential cross sections (TDCS) produced by nonperturbative $[4,7]$ and perturbative, with respect to the field, $[11,12]$ calculations. Less detailed double differential cross section (DDCS), integrated over the escape angle of one of the photoelectrons, was not explored so far. The only report of DDCS by Barna et al. [13] is concerned with the sequential regime of TPDI which shows much less sensitivity to many-electron correlations.

In the meantime, DDCS is much more appealing for experimental studies because of a larger volume of phase space being probed and, hence, significantly improved statistics. It can bring a wealth of information as was demonstrated by Knapp et al. [14] who were able to identify various mechanisms of single-photon double ionization (SPDI) of He. Similar angular distributions differential with respect to the sum or difference momenta of the photoelectron pair can also be readily obtained [15-17]. They proved to be useful in pinpointing propensity rules which govern the two-electron escape in the Jacobian coordinates [18].

\footnotetext{
*a.kheifets@anu.edu.au

${ }^{\dagger}$ On leave from the Institute of Spectroscopy, Russian Academy of Sciences.
}

In this paper, we bridge the gap between the total integrated and fully differential cross sections of TPDI of He and present the calculations of DDCS in conventional and Jacobian coordinates. The latter calculation allows us to obtain the recoil ion momentum distribution which can be compared directly with a recent FLASH experiment [19]. We use essentially the same dynamical model as was applied in our previous works on TPDI of $\mathrm{He}[11,20]$. In this model, the atom-field interaction is restricted to the lowest second-order perturbation theory which is further simplified by the closure approximation. In the meantime, the electron-electron interaction is treated nonperturbatively by the convergent-closecoupling (CCC) method and included in full. In the closure approximation, all the intermediate states of the target atom between absorption of the first and second photons are weighted equally and summed over with an average energy denominator. The closure approximation restricts the ability of this model to provide accurate absolute cross sections as it underestimates the contribution of the intermediate states with a small energy denominator. However, due to the long range of the Coulomb force, the angular correlation pattern in the two-electron continuum is formed at large distances (and times) when the field pulse is long gone. It shows, therefore, little sensitivity to the details of the atom-field interaction. And indeed, the angular correlation patterns obtained with the present model [11] are remarkably similar to nonperturbative, with respect to the field, time-dependent close-coupling calculations $[4,7]$. We thus believe that this model should be adequate for DDCS calculations.

We start our derivation from the TPDI TDCS differential with respect to the photoelectron angles and energy,

$$
\begin{aligned}
& \frac{d^{3} \sigma_{M}}{d \Omega_{1} d \Omega_{2} d E_{2}} \propto \mid \sum_{\substack{L=0,2 \\
l_{1} m_{1} \\
l_{2} m_{2}}} \underset{l_{2} l_{1} m_{1}}{L M} Y_{l_{1} m_{1}}\left(\hat{\boldsymbol{k}}_{1}\right) Y_{l_{2} m_{2}}\left(\hat{\boldsymbol{k}}_{2}\right) \\
& \times\left.(-i)^{l_{1}+l_{2}} e^{i\left(\delta_{l_{1}}+\delta_{l_{2}}\right)} D_{l_{1} l_{2}}^{L}\left(E_{1}, E_{2}\right)\right|^{2} .
\end{aligned}
$$

Here the index $M$ refers to the polarization state of light, $M=0$ and 2 correspond to linear and circular polarization, 
respectively. In the latter case, only the quadrupole channel $L=2$ contributes to TDCS. The radial matrix elements $D_{l_{1} l_{2}}^{L}\left(E_{1}, E_{2}\right)$ are obtained in the simplified second-order perturbation theory as described in earlier work [11]. By integrating Eq. (1) over $d \Omega_{1}$, we get DDCS which can be presented in the form

$$
\frac{d^{2} \sigma}{d \Omega_{2} d E_{2}} \propto \sum_{J=0,2,4} \beta_{J} P_{J}\left(\cos \theta_{2}\right),
$$

where the $\beta$ coefficients are given by the following expression:

$$
\begin{aligned}
\beta_{J}= & \hat{J}^{2} \sum_{l_{1}} \sum_{l_{2} l_{2}^{\prime}} \hat{L} \hat{L}^{\prime} \hat{l_{2}} \hat{l}_{2}^{\prime}(-1)^{l_{1}+J}\left(\begin{array}{ccc}
L & J & L^{\prime} \\
M & 0 & -M
\end{array}\right)\left(\begin{array}{ccc}
l_{2} & J & l_{2}^{\prime} \\
0 & 0 & 0
\end{array}\right) \\
& \times\left\{\begin{array}{ccc}
L L^{\prime} & J & L^{\prime} \\
l_{2}^{\prime} & l_{1} & l_{2}
\end{array}\right\} \operatorname{Re}\left[(-i)^{l_{2}-l_{2}} e^{i\left(\delta_{l_{2}}-\delta_{l_{2}^{\prime}}\right)} D_{l_{1} l_{2}}^{L}\left(D_{l_{1} l_{2}^{\prime}}^{L^{\prime}}\right)^{*}\right] .
\end{aligned}
$$

Here we introduced the hat symbol $\hat{L}=(2 L+1)^{1 / 2}$ and dropped the explicit energy dependence of the matrix elements $D_{l_{1} l_{2}}^{L}\left(E_{1}, E_{2}\right)$ for brevity. In deriving Eqs. (2) and (3), we coupled the angular momenta of the photoelectron pair $L, L^{\prime}$ into $J$ using the graphical angular momentum projection summation technique [21]. Equation (3) coincides with an analogous expression for $\beta$ parameters of two-photon single ionization $[22,23]$ in which the role of $l_{1}$ is assumed by the angular momentum of the bound target electron. In the following, we divide the $\beta$ parameters by the single differential, with respect to energy $E_{2}$, cross section such that $\beta_{0}=1$ and write the DDCS as

$$
\frac{d^{2} \sigma}{d \Omega_{2} d E_{2}}=\frac{d \sigma}{d E_{2}} \frac{1}{4 \pi}\left[1+\beta_{2} P_{2}\left(\cos \theta_{2}\right)+\beta_{4} P_{4}\left(\cos \theta_{2}\right)\right] .
$$

In comparison, the DDCS of SPDI contains only the $\beta_{2}$ term which is given by the same Eq. (3) with $L=L^{\prime}=1$.

To derive DDCS in the Jacobian coordinates, we use the method proposed by Pont and Shakeshaft [24] for SPDI. We start from the TPDI amplitude in the form suggested by Istomin et al. [12],

$$
\begin{aligned}
F\left(\boldsymbol{k}_{1}, \boldsymbol{k}_{2}\right)= & f_{1}\left(\hat{\boldsymbol{k}}_{1} \cdot \boldsymbol{e}\right)^{2}+f_{2}\left(\hat{\boldsymbol{k}}_{2} \cdot \boldsymbol{e}\right)^{2}+f_{s}\left(\hat{\boldsymbol{k}}_{1} \cdot \boldsymbol{e}\right)\left(\hat{\boldsymbol{k}}_{2} \cdot \boldsymbol{e}\right) \\
& +f_{0}(\boldsymbol{e} \cdot \boldsymbol{e}) .
\end{aligned}
$$

Here $\hat{\boldsymbol{k}}_{i}=\boldsymbol{k}_{i} / k_{i}, i=1,2$ are the unit vectors directed along the photoelectron momenta. The amplitudes $f_{i}, i=0,1,2, s$ depend on the essential dynamic variables $E_{1}, E_{2}$, and $\cos \theta_{12}=\boldsymbol{k}_{1} \cdot \boldsymbol{k}_{2} /\left(k_{1} k_{2}\right)$. We rewrite Eq. (5) with respect to the center-of-mass momentum of the two-electron subsystem $p=k_{1}+k_{2}$ and the relative momentum $k=k_{1}-k_{2}$,

$$
F(\boldsymbol{p}, \boldsymbol{k})=f_{p}(\hat{\boldsymbol{p}} \cdot \boldsymbol{e})^{2}+f_{k}(\hat{\boldsymbol{k}} \cdot \boldsymbol{e})^{2}+f_{k p}(\hat{\boldsymbol{k}} \cdot \boldsymbol{e})(\hat{\boldsymbol{p}} \cdot \boldsymbol{e})+f_{0}(\boldsymbol{e} \cdot \boldsymbol{e}),
$$

where

$$
f_{p}=\frac{p^{2}}{4}\left(\frac{f_{1}}{k_{1}^{2}}+\frac{f_{2}}{k_{2}^{2}}+\frac{f_{s}}{k_{1} k_{2}}\right) \approx \frac{p^{2}}{4}\left[f\left(\frac{1}{k_{1}^{2}}+\frac{1}{k_{2}^{2}}\right)+\frac{f_{s}}{k_{1} k_{2}}\right],
$$

$$
\begin{gathered}
f_{k}=\frac{k^{2}}{4}\left(\frac{f_{1}}{k_{1}^{2}}+\frac{f_{2}}{k_{2}^{2}}-\frac{f_{s}}{k_{1} k_{2}}\right) \approx \frac{k^{2}}{4}\left[f\left(\frac{1}{k_{1}^{2}}+\frac{1}{k_{2}^{2}}\right)-\frac{f_{s}}{k_{1} k_{2}}\right], \\
f_{k p}=\frac{k p}{2}\left(\frac{f_{1}}{k_{1}^{2}}-\frac{f_{2}}{k_{2}^{2}}\right) \approx \frac{k p}{2} f\left(\frac{1}{k_{1}^{2}}-\frac{1}{k_{2}^{2}}\right) .
\end{gathered}
$$

Here we used a weak asymmetry approximation $f_{1} \approx f_{2} \equiv f$ thus neglecting the energy sharing dependence of the amplitudes. The accuracy of this approximation for SPDI was tested by Pont and Shakeshaft [24] who found it adequate for qualitative interpretation of the recoil ion momentum distribution. Although we do not perform such a test in the TPDI case, the calculations of $\mathrm{Hu}$ et al. [7] demonstrated little influence of the energy sharing on the angular distribution of photoelectrons thus implicitly validating the weak asymmetry approximation.

Directing the (linear) polarization axis along $z$ and noting that $\boldsymbol{p} \cdot \boldsymbol{e}=p \cos \theta_{p}$ and $\boldsymbol{k} \cdot \boldsymbol{e}=k \cos \theta_{k}$ we write squared amplitude (6) as

$$
|F(\boldsymbol{p}, \boldsymbol{k})|^{2}=\sum_{i, j} \operatorname{Re}\left\{f_{i} f_{j}^{*}\right\} \cos ^{N p_{i}+N p_{j}} \theta_{p} \cos ^{N k_{i}+N k_{j}} \theta_{k},
$$

where $i, j=p, k, k p, 0$ and $N p_{p}=N k_{k}=2, N p_{k p}=N k_{k p}=1, N p_{k}$ $=N k_{p}=N p_{0}=N k_{0}=0$. The next step is to expand the amplitude factors entering Eq. (7) into the Legendre polynomial series,

$$
\operatorname{Re}\left\{f_{i} f_{j}^{*}\right\}=\sum_{l}(2 l+1) P_{l}(\hat{\boldsymbol{k}} \cdot \hat{\boldsymbol{p}}) f_{i j}^{l} .
$$

With the help of the linear transformation $\cos ^{n} \theta_{k}$ $=\sum_{l=0}^{n} C_{l} P_{l}(\hat{\boldsymbol{k}} \cdot \boldsymbol{e})$ and the Legendre polynomials spherical integral formula $\int d \Omega_{k} P_{l}(\hat{\boldsymbol{k}} \cdot \boldsymbol{e}) P_{l^{\prime}}(\hat{\boldsymbol{k}} \cdot \hat{\boldsymbol{p}})=4 \pi(2 l+1)^{-1} P_{l}(\hat{\boldsymbol{p}} \cdot \boldsymbol{e}) \delta_{l l^{\prime}}$, we can perform the spherical integration to obtain

$$
\frac{1}{4 \pi} \int d \Omega_{k}|F(\boldsymbol{p}, \boldsymbol{k})|^{2}=A_{0}+A_{2} P_{2}\left(\cos \theta_{p}\right)+A_{4} P_{4}\left(\cos \theta_{p}\right),
$$

where the coefficients $A_{0}, A_{2}$, and $A_{4}$ are the linear combination of the coefficients $f_{i j}^{l}$. The explicit expressions are cumbersome but their derivation can be greatly assisted by Mathematica. The same spherical integration technique can be applied directly to Eq. (5) thus giving an alternative method of obtaining the $\beta$ coefficients in Eq. (4). We used this equivalence as a check of the accuracy of our computations.

The DDCS is the spherical integral (8) multiplied by an extra kinematical factor $k p$ [24],

$$
\begin{aligned}
\frac{d^{2} \sigma}{d \Omega_{p} d E_{p}} & \propto k p \frac{1}{4 \pi} \int d \Omega_{k}|F(\boldsymbol{p}, \boldsymbol{k})|^{2} \\
& =\frac{d \sigma}{d E_{p}} \frac{1}{4 \pi}\left[1+\beta_{2} P_{2}\left(\cos \theta_{p}\right)+\beta_{4} P_{4}\left(\cos \theta_{p}\right)\right],
\end{aligned}
$$

where 

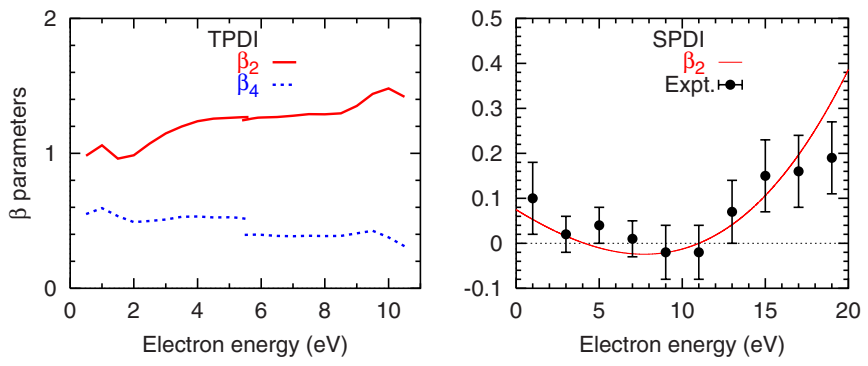

FIG. 1. (Color online) Angular anisotropy $\beta$ parameters for twophoton (left-hand side) and single-photon (right-hand side) double ionization of $\mathrm{He}$ at the excess energies of 11 and $20 \mathrm{eV}$, respectively. Experimental data for SPDI are from Bräuning et al. [25].

$$
\frac{1}{4 \pi} \frac{d \sigma}{d E_{p}} \propto k p A_{0}, \quad \beta_{2}=\frac{A_{2}}{A_{0}}, \quad \beta_{4}=\frac{A_{4}}{A_{0}} .
$$

We note that $d \sigma / d E_{p}=0$ for $p=0$ even though $A_{0} \neq 0$ as the antiparallel escape resulting in $p=0$ is not forbidden in TPDI as opposed to SPDI.

Now we turn to results of our calculations. In the lefthand panel of Fig. 1 we show the $\beta$ parameters in Eq. (4) for TPDI of He with linearly polarized light at the excess energy of $11 \mathrm{eV}$ above the double ionization threshold which corresponds to the photon energy $\omega=45 \mathrm{eV}$. For comparison, in the right-hand panel of the same figure, we show the $\beta$ parameter for SPDI at the excess energy of $20 \mathrm{eV}$ (the photon energy $\omega=99 \mathrm{eV}$ ). The experimental data for SPDI are from Bräuning et al. [25].

In the CCC method, the electrons are explicitly distinguishable. The label 1 is attached to the slow electron which is described as a positive energy pseudostate whereas the label 2 corresponds to the fast electron which is considered as a Coulomb wave. Thus the expressions (3) and (4) define the angular distribution of the fast photoelectron $E_{2}>E / 2$ where $E$ is the excess energy above the double ionization threshold. To obtain the angular distribution of the slow photoelectron, we rely on the exchange symmetry of the matrix elements $D_{l_{1} l_{2}}^{L}\left(E_{1}, E_{2}\right)=D_{l_{2} l_{1}}^{L}\left(E_{2}, E_{1}\right)$. Since the slow and fast electrons are described differently, numerical values of the direct and exchange matrix elements might be somewhat different. This is reflected in a gap of the $\beta$ parameters at $E / 2$.

By comparing the left-hand and right-hand panels of Fig. 1 , we observe a significant qualitative difference between the $\beta$ parameters for TPDI and SPDI. In the two-photon case, both $\beta_{2}$ and $\beta_{4}$ are large and positive. In contrast, in the single-photon case, the $\beta_{2}$ parameter varies widely from nearly zero for a very slow photoelectron to large positive values for a fast photoelectron. This tendency to large $\beta_{2}$ is exemplified at larger excess energies. For instance, at $100 \mathrm{eV}$ excess energy, $\beta_{2} \approx 1.4$ for the $99 \mathrm{eV}$ photoelectron which demonstrates a strongly dipolar angular distribution [17]. At larger still excess energy of $450 \mathrm{eV}, \beta_{2} \approx 2$ at the top end of the excess energy scale which corresponds to the complete alignment of the fast photoelectron with the polarization axis of light [14]. This energy dependence of $\beta_{2}$ can be interpreted as a gradual cross over between two different regimes of SPDI. Nearly zero $\beta_{2}$ parameter of a slow photoelectron
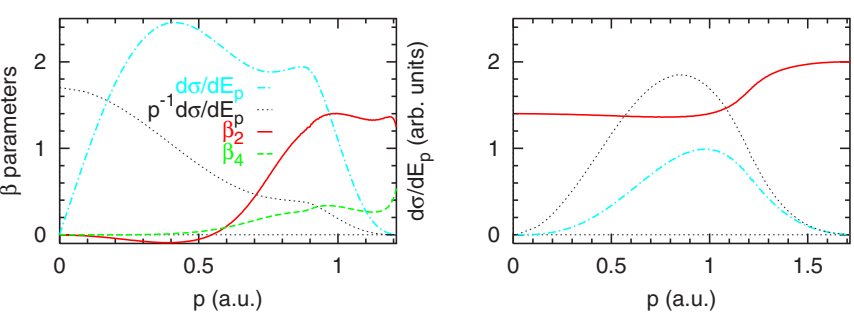

FIG. 2. (Color online) Angular anisotropy $\beta$ parameters and energy distribution $d \sigma / d E_{p}$ of the recoil ion for single-photon (right-hand side) and two-photon (left-hand side) double ionization of $\mathrm{He}$ at the excess energies of 11 and $20 \mathrm{eV}$, respectively.

corresponds to a fully isotropic angular distribution which is a footprint of the shake-off mechanism. On the contrary, a large positive $\beta_{2}$ parameter for a fast photoelectron corresponds to a direct knock out when the whole of the photon energy and angular momentum is absorbed by the fast photoelectron.

This can be seen from the explicit expression of the $\beta_{2}$ parameter for SPDI obtained from Eq. (3) by setting $L=L^{\prime}=1$. When the shake off is the main mechanism of the SPDI, the slow electron emerges predominantly in the $s$ state. One therefore can truncate the sum in (3) by a single term $l_{2}=l_{2}^{\prime}=0$ which leads immediately to $\beta_{2}=0$. To find the $\beta_{2}$ parameter for a fast photoelectron, one can employ the exchange symmetry $D_{l_{1} l_{2}}\left(E_{1}, E_{2}\right)=D_{l_{2} l_{1}}\left(E_{2}, E_{1}\right)$ to establish the only surviving term corresponding to $l_{1}=0, l_{2}=1$, and $\beta=2$.

In TPDI, because the target atom interacts with the field twice, the shake-off electron can be ejected in a $p$ wave after first being promoted from the ground $1 s$ state to an excited $n p$ state. In this scenario, the main contribution to the angular distribution of the slow electron comes from the $l_{1}=l_{2}=1$ term which leads, in the dominant $L=2$ channel, to $\beta_{2}=1$ and $\beta_{4}=0$. This is indeed close to the calculated value of $\beta_{2}$ and explains much smaller values of $\beta_{4}$. By way of the exchange symmetry, one can argue that the same angular terms would be dominant for the fast electron which explains a rather weak energy dependence of $\beta$ parameters. A nonzero value of $\beta_{4}$ indicates that the slow electron can also emerge in a $d$ state as a result of two repeated knock-out processes. Most likely, as in the case of SPDI, a clear separation of the shake-off and knock-out ionization mechanisms would occur at much higher photon energies. However, at these energies, the sequential regime of TPDI would become dominant.

Now we turn our discussion to the angular distribution of the recoil ion momentum $\boldsymbol{K}$ which is defined by the angular asymmetry parameters for the sum momentum $\boldsymbol{p}=\boldsymbol{k}_{1}+\boldsymbol{k}_{2}=-\boldsymbol{K}$. In the left-hand panel of Fig. 2 we plot the angular asymmetry $\beta$ parameters and the energy distribution $d \sigma / d E_{p}$ corresponding to the sum momentum $\boldsymbol{p}$ for the TPDI of He at the excess energy of $11 \mathrm{eV}$. For comparison, in the right-hand panel of Fig. 2, we plot the analogous parameters for the SPDI at the excess energy of $20 \mathrm{eV}$. Again, as in Fig. 1 , there is a qualitative difference between the TPDI and SPDI cases. In the two-photon case, the $\beta$ parameters change from nearly zero to large positive values as $p$ varies from 0 to $p_{\max }$. This is to be compared with the large and positive $\beta_{2}$ 
parameter which varies very little in SPDI. The energy distribution $d \sigma / d E_{p}$ is zero both for $p=0$ and $p=p_{\max }(k=0)$ due to the kinematic factor $k p$. However, the function $p^{-1} d \sigma / d E_{p}$ is large at $p=0$ and decays monotonously towards $p_{\max }$ in TPDI but has a broad maximum at about $\simeq p_{\max } / 2$ in SPDI.

This qualitatively different behavior of $\beta$ parameters can be explained in the following way. Inspection of Eq. (7) shows that the terms containing various powers of $\cos \theta_{p}$ and thus contributing to $A_{2}$ and $A_{4}$ coefficients of (8) are all vanishing when $p \rightarrow 0$. In the meantime, $A_{0}$ remains finite as $f_{k}$ is nonvanishing in this limit. This results in zero values of $\beta_{2}$ and $\beta_{4}$ and a finite value of $p^{-1} d \sigma / d E_{p}$ at $p=0$. In comparison, the SPDI amplitude has the form $F(\boldsymbol{p}, \boldsymbol{k})$ $=f_{p}(\boldsymbol{p} \cdot \boldsymbol{e})+f_{k}(\boldsymbol{k} \cdot \boldsymbol{e})$, where $f_{p, k}=0.5\left(f_{1} / k_{1} \pm f_{2} / k_{2}\right)$. When $p \rightarrow 0, f_{k}$ goes to zero as well and both the coefficients $A_{0}$ and $A_{2}$ become small. This results in a finite $\beta_{2}$ and vanishing $p^{-1} d \sigma / d E_{p}$ at $p=0$. We note that this analysis reflects the tensorial structure of the SPDI and TPDI amplitudes and thus remains valid for any atom other than He.

This different behavior of double ionization amplitudes in the single- and two-photon case has a profound implication when the recoil ion momentum distribution is measured experimentally. In a typical cold target recoil ion momentum spectroscopy (COLTRIMS) experiment, the following quantity is measured [17]:

$$
\frac{d \sigma}{d p_{x} d p_{z}}=\int_{-\Delta p_{y}}^{\Delta p_{y}} d p_{y} \frac{1}{p} \frac{d \sigma}{d E_{p} d \Omega_{p}} .
$$

Here the $3 \mathrm{D}$ momentum is projected on the polarization plane by way of integration over the momentum component $p_{y}$ in the direction of the photon propagation. The limits of integration $\Delta p_{y}$ are chosen depending on the signal count rate. For SPDI, thus observed momentum distribution will peak at $\simeq p_{\max } / 2$ where $\beta_{2}$ is large and positive resulting in a broad dipole structure aligned along the polarization axis of light as is indeed the case for various excess energies ranging from 1 to $100 \mathrm{eV}$ [15-17]. On the contrary, in the TPDI case, the intensity of the momentum distribution is largest near the origin where the $\beta$ parameters are close to zero. Hence, there will be very little anisotropy seen in the recoil ion momentum distribution except for large momenta where the experimental signal is fairly weak. A recent COLTRIMS study of TPDI of Ne at photon energy of $38.8 \mathrm{eV}$ confirmed this prediction [19]. Preliminary results on $\mathrm{He}$ at $45 \mathrm{eV}$ photon energy seem to also fit into this pattern [26].

The authors wish to thank Robert Moshammer for providing experimental results. The authors acknowledge support of the Australian Research Council in the form of Discovery Grant No. DP0771312. Facilities of the Australian Partnership for Advanced Computing were used.
[1] M. S. Pindzola and F. Robicheaux, J. Phys. B 31, L823 (1998).

[2] L. A. A. Nikolopoulos and P. Lambropoulos, J. Phys. B 34, 545 (2001).

[3] T. Mercouris, C. Haritos, and C. A. Nicolaides, J. Phys. B 34, 3789 (2001).

[4] J. Colgan and M. S. Pindzola, Phys. Rev. Lett. 88, 173002 (2002).

[5] L. Feng and H. W. van der Hart, J. Phys. B 36, L1 (2003).

[6] B. Piraux, L. Bauer, S. Laulan, and H. Bachau, Eur. Phys. J. D 26, 7 (2003).

[7] S. X. Hu, J. Colgan, and L. A. Collins, J. Phys. B 38, L35 (2005).

[8] E. Foumouo, G. L. Kamta, G. Edah, and B. Piraux, Phys. Rev. A 74, 063409 (2006).

[9] I. A. Ivanov and A. S. Kheifets, Phys. Rev. A 75, 033411 (2007).

[10] L. A. A. Nikolopoulos and P. Lambropoulos, J. Phys. B 40, 1347 (2007).

[11] A. S. Kheifets and I. A. Ivanov, J. Phys. B 38, 471 (2006).

[12] A. Y. Istomin, E. A. Pronin, N. L. Manakov, S. I. Marmo, and
A. F. Starace, Phys. Rev. Lett. 97, 123002 (2006).

[13] I. F. Barna, J. Wang, and J. Burgdorfer, Phys. Rev. A 73, 023402 (2006).

[14] A. Knapp et al., Phys. Rev. Lett. 89, 033004 (2002).

[15] R. Dörner et al., Phys. Rev. Lett. 77, 1024 (1996).

[16] H. Bräuning et al., J. Phys. B 30, L649 (1997).

[17] A. Knapp, J. Phys. B 35, L521 (2002).

[18] M. Walter, J. S. Briggs, and J. M. Feagin, J. Phys. B 33, 2907 (2000).

[19] R. Moshammer et al., Phys. Rev. Lett. 98, 203001 (2007).

[20] A. S. Kheifets, I. A. Ivanov, and I. Bray, Phys. Rev. A 75, 024702 (2007)

[21] D. A. Varshalovich, Quantum Theory of Angular Momentum (World Scientific, Philadelphia, 1988).

[22] C.-R. Liu, B. Gao, and A. F. Starace, Phys. Rev. A 46, 5985 (1992).

[23] G. F. Gribakin et al., J. Phys. B 32, 5463 (1999).

[24] M. Pont and R. Shakeshaft, Phys. Rev. A 54, 1448 (1996).

[25] H. Bräuning et al., J. Phys. B 30, L649 (1997).

[26] R. Moshammer (private communication). 